\title{
A BOUND ON THE $p$-LENGTH OF P-SOLVABLE GROUPS
}

\author{
JON GONZÁLEZ-SÁNCHEZ \\ Departamento de Matemáticas, Facultad de Ciencias, Universidad del País Vasco-Euskal Herriko \\ Unibertsitatea, Spain \\ email: jon.gonzalez@ehu.es
}

\author{
and FRANCESCA SPAGNUOLO \\ Departament dÀlgebra, Universitat de València; Dr. Moliner, 50, 46100, Burjassot, València, Spain. \\ email: francesca.spagnuolo@uv.es
}

(Received 21 February 2013; accepted 4 November 2013; first published online 26 August 2014)

\begin{abstract}
Let $G$ be a finite $p$-solvable group and $P$ a Sylow $p$-subgroup of $G$. Suppose that $\gamma_{\ell(p-1)}(P) \subseteq \gamma_{r}(P)^{p^{s}}$ for $\ell(p-1)<r+s(p-1)$, then the $p$-length is bounded by a function depending on $\ell$.
\end{abstract}

1991 Mathematics Subject Classification. 20D10 (20D15)

1. Introduction. All the groups considered are finite. In the following, $p$ will be a prime number.

A group $G$ is $p$-solvable if it has a sequence of subgroups

$$
G=N_{1} \supset N_{2} \supset \ldots \supset N_{k}=1
$$

such that $N_{i+1}$ is a normal subgroup of $N_{i}$ and the index $\left|N_{i}: N_{i+1}\right|$ is either coprime to $p$ or a power of $p$. In such a case the minimal number of factors $N_{i} / N_{i+1}$, which are $p$-groups, is called the $p$-length of $G$. Alternatively, a group $G$ is $p$-solvable if the upper series

$$
1 \subseteq O_{p^{\prime}}(G) \subseteq O_{p^{\prime}, p}(G) \subseteq O_{p^{\prime}, p, p^{\prime}}(G) \subseteq \ldots
$$

ends in $G$ and we call the $p$-length of $G$ to the number of symbols $p$ appearing in the series. Recall that the previous series is constructed as follows: $O_{p^{\prime}}(G)$ is the maximal normal $p^{\prime}$-subgroup of $G ; O_{p^{\prime}, p}(G)$ denotes the inverse image of the maximal normal $p$-subgroup of $G / O_{p^{\prime}}(G) ; O_{p^{\prime}, p, p^{\prime}}(G)$ denotes the inverse image of the maximal normal $p^{\prime}$-subgroup of $G / O_{p^{\prime}, p}(G)$ and so on.

Much is known about the $p$-length of $p$-solvable groups. For example, by a result of Hall and Higman [4], explicit bounds of the $p$-length are known in terms of the derived length or the exponent of the Sylow $p$-subgroup. More recently González-Sánchez and Weigel [2] proved that if $p$ is odd and the elements of order $p$ of the Sylow subgroups are contained in the ( $p$-2)-centre of the Sylow p-subgroup, then the $p$-length is equal to 1. This result was generalised by Khukhro in [5] by proving that if the elements of order $p$ (or 4 if $p=2$ ) of the Sylow $p$-subgroup are contained in the $\ell$-centre of the Sylow $p$-subgroup, then the $p$-length of the group is bounded in terms of $\ell$. In the same paper, Khukhro [5] also proved that if the Sylow $p$-subgroup is powerful, then the $p$-length is 1 , and he posted the question on whether this result can be generalised in the same 
way as the result for the Sylow $p$-central subgroup. In this short paper we will try to answer this question by giving a bound on the $p$-length of a $p$-solvable group in terms of some power-commutator conditions in the Sylow $p$-subgroup.

MAIN TheOREM. Let $G$ be a p-solvable group and $P$ a Sylow p-subgroup of $G$. Suppose that

$$
\gamma_{\ell(p-1)}(P) \subseteq \gamma_{r}(P)^{p^{s}}
$$

for $\ell(p-1)<r+s(p-1)$. Then the p-length of $G$ is bounded in terms of $l$. This condition holds in particular when $\gamma_{\ell(p-1)}(P) \subseteq P^{p^{l}}$.

The notation is standard in the group theory. The subgroups $O_{p}(G)$ and $O_{p^{\prime}}(G)$ denote the maximal normal subgroup of order a power of $p$ and coprime to $p$ respectively. $\left[N,{ }_{k} M\right]$ denotes the commutator subgroup $[N, M, \ldots, M]$, where $M$ appears $k$ times.

Consider now a group $G$ and a normal elementary abelian $p$-group $V$. Let $\varphi$ be the action by conjugation of $G$ on $V$ :

$$
\varphi: G \times V \rightarrow V /(g, v) \rightarrow v^{g} .
$$

We can regard $V$ as a vector space over the field $\mathbb{F}_{p}$. In this case, the action by conjugation of $G$ on $V$ can be regarded as an action by linear transformation on this vector space, and we denote this action as follows:

$$
(g, v) \in G \times V \rightarrow v T(g),
$$

where $T(g)$ denotes the action of an element $g$ of $G$ on $V$. If we denote by $1_{V}$ the identity transformation on $V$, then

$$
v\left(T(g)-1_{V}\right)=[v, g] .
$$

2. Potent filtrations. Let $P$ be a $p$-group, we say that a sequence of normal subgroups $\left(N_{i}\right)_{i=1}^{k}$ is a potent filtration of type $\ell$ of $P$ if

(1) $N_{i} \subseteq N_{j}$, for all $i>j$,

(2) $N_{k}=1$,

(3) $\left[N_{i}, P\right] \subseteq N_{i+1}$, for $i=1, \ldots, k-1$,

(3) $\left[N_{i}, l P\right] \subseteq N_{i+1}^{p}$, for $i=1, \ldots, k-1$.

Furthermore, we say that a group $N$ is $P F$-embedded of type $\ell$ in $P$ if there is a potent filtration of $P$ beginning at a subgroup $N$.

Proposition 1. Let $P$ be a pro-p group, and let $\left\{N_{i}\right\}_{i=1}^{k}$ a potent filtration of $P$ of type $\ell$. Then:

(1) $\left[N_{i}^{p}, P\right]=\left[N_{i}, P\right]^{p}$ for all $i$,

(2) $\left[N_{i}, P\right]_{i=1, \ldots k}$ is a potent filtration of type $\ell$ of $P$,

(3) $N_{i}^{p}$ is a potent filtration of type $\ell$ of $P$.

Proof. See [1, Proposition 3.2].

We continue with the case where $N$ is PF-embedded subgroup of type $p-2$. 
Proposition 2. Let $G$ be a p-solvable group and $P$ a Sylow p subgroup of G. Suppose that $N$ is a PF-embedded subgroup of type $p-2$ of $P$. Then $N \subseteq O_{p^{\prime} p}(G)$.

Proof. We can assume that $O_{p^{\prime}}(G)=1$. In order to simplify notations, we put $O_{p}(G)=V$. Modding out by the Frattini subgroup, we can also assume that $V$ is an elementary abelian $p$-group. Furthermore, $V=C_{G}(V)$ [3, Theorem 6.3.2]. Therefore, $G / V$ acts faithfully on $V$; so we can regard $V$ as a $\mathbb{F}_{p}(G / V)$-module. Moreover, we can consider the Jordan-Holder series of $V$ as an $\mathbb{F}_{p}[G / V]$-module:

$$
V=V_{1} \supseteq V_{2} \supseteq V_{3} \supseteq \ldots \supseteq V_{n}
$$

such that $V_{i} / V_{i+1}$ is simple for every $i$. Without loss of generality, we can take the quotient of the $\mathbb{F}_{p}[G / V]$-module $V$ over the second term of Jordan-Holder series and assume that $V$ is a simple $\mathbb{F}_{p}[G / V]$-module.

Take $\left(N_{i}\right)_{i=1}^{k}$ as a potent filtration starting at $N$. We will prove by reverse induction on $i$ that for all $i, N_{i} \subseteq V$. For $i$ large enough it is clear. Suppose now that $N_{i+1} \subseteq V$. Take $v \in V$ and $n \in N_{i}$. Then

$$
[v, \underbrace{n, \ldots, n}_{p-2}] \in[V, \underbrace{N_{i}, \ldots N_{i}}_{p-2}] \subseteq\left[N_{i, p-2} P\right] \subseteq N_{i}^{p} \subseteq V^{p}=1 .
$$

Therefore, for all $v \in V$ and $n \in N_{i}$, we have

$$
v\left(T(n)-1_{V}\right)^{p-2}=0 .
$$

By [2, Corollary 5.2] the size of Jordan blocks of $T(n)$ can only be 1. Therefore $N_{i} \subseteq \operatorname{ker}(T)$ and by [3, Theorem 6.3.2] $N_{i} \subseteq C_{G}(V)=V$.

In order to prove the Main Theorem, we will need a weaker result for PF-embedded subgroups of type $p-1$.

Proposition 3. Let $G$ be a p-solvable group, $P$ a Sylow $p$ subgroup of $G$ and $N a$ $P F$-embedded subgroup of type $p-1$ of $P$. Then,

(a) If $p \geq 5$, then $N^{p} \subseteq O_{p^{\prime} p}(G)$.

(b) If $p=3$, then $N^{p^{2}} \subseteq O_{p^{\prime} p}(G)$.

(c) If $p=2$, then $N \subseteq O_{p^{\prime} p}(G)$.

Proof. As in the proof of Proposition 3, we can assume that $O_{p^{\prime}}(G)=1$ and $O_{p}(G)=V$ is a simple $\mathbb{F}_{p}(G / V)$-module. Note that $V=C_{G}(V)$ (see [3, Theorem 6.3.2]). Take $\left(N_{i}\right)_{i=1}^{k}$ as a potent filtration starting at $N$.

(a) We will prove by reverse induction on $i$ that $N_{i}^{p} \subseteq V$. For $i$ large enough it is clear. Let us suppose that $N_{i+1}^{p} \subseteq V$. Take $n^{p} \in N_{i}^{p}$ and $v \in V$. Then by Proposition 1 ,

$$
\left[v, n^{p}, n^{p}\right] \in\left[V, N_{i}^{p}, N_{i}^{p}\right] \subseteq\left[P, N_{i}, N_{i}\right]^{p^{2}} \subseteq N_{i+1}^{p^{2}} \subseteq V^{p}=1 .
$$

Therefore,

$$
v\left(T\left(n^{p}\right)-1_{V}\right)^{2}=1 .
$$

Then, as in Proposition 3 and since $p \geq 5$, the Jordan block size can only be 1 . So $N_{i}^{p} \subseteq C_{G}(V)=V$. 
(b) As in (a), we prove by reverse induction on $i$ that $N_{i}^{p^{2}} \subseteq V$. For $i$ large enough it is clear. Let us suppose that $N_{i+1}^{p^{2}} \subseteq V$. Take $n^{p^{2}} \in N_{i}^{p^{2}}$ and $v \in V$. Then, by [1, Theorem 2.4],

$$
\left[v, n^{p^{2}}\right] \in\left[V, N_{i}^{p^{2}}\right] \subseteq\left[V,{ }_{p^{2}} N_{i}\right] \subseteq N_{i+1}^{p^{3}} \subseteq V^{p}=1
$$

Therefore, $N_{i}^{p^{2}} \subseteq C_{G}(V)=V$.

(c) As in (a) we prove by reverse induction on $i$ that $N_{i} \subseteq V$. For $i$ large enough, it is clear. Let us suppose that $N_{i+1} \subseteq V$. Take $n \in N_{i}$ and $v \in V$. Then,

$$
[n, v] \in\left[N_{i}, V\right] \subseteq N_{i+1}^{2} \subseteq V^{p}=1 .
$$

Therefore, $n \in C_{G}(V)=V$ and $N_{i} \subseteq V$.

3. Proof of the main theorem. Now we introduce a family $E_{k, r}(P)$ of subgroups of a finite $p$-group $P$ as in [1].

Definition 4. Let $P$ be a finite $p$-group. For any pair $k, r$ of positive integers, we define the subgroup

$$
E_{k, r}(P)=\prod_{i+j(p-1) \geq k \text { and } i \geq r} \gamma_{i}(P)^{p^{j}}
$$

The next theorem is a stronger version of the Main Theorem.

THeORem 5. Let $G$ be a p-solvable group, and let $P$ be a Sylow p-subgroup of $G$. Suppose that $\gamma_{\ell(p-1)}(P) \subseteq E_{\ell(p-1)+1,1}(P)$, then the p-length of $G$ is bounded in terms of $\ell$.

Proof. Put $E=E_{(\ell-1)(p-1), 1}(P)$. By [1, Theorem 4.8], the subgroup $E$ is PFembedded of type $p-1$ in P. So $E^{p^{2}} \subseteq O_{p^{\prime}, p}(G)$.

By construction, the exponent of $P / O_{p^{\prime}, p}(G)$ is at most the exponent of $P / E^{p^{2}}$ which is bounded by $p^{\ell+1}$. Therefore, by [4, Theorem A], the $p$-length of $G$ is bounded in terms of $\ell$.

4. A question and a lemma. In Section 2, we proved that if $G$ is a 2-solvable group, $P$ is a Sylow 2-subgroup of $P$ and $N$ is a PF-embedded subgroup of $P$, then $N \subseteq O_{p, p^{\prime}}(G)$. For the case were $p$ is an odd prime we have only a weaker version of the result. Therefore, the following question arises naturally.

Question 1. Let $G$ be a $p$-solvable group, $P$ is a Sylow $p$-subgroup of $P$ and $N$ is a PF-embedded subgroup of $P$. Then $N \subseteq O_{p, p^{\prime}}(G)$.

A positive answer to this question will provide an improvement of the implicit bound in Theorem 5. The following lemma could be helpful. It is a generalisation of [3, Theorem 6.3.2].

Lemma 6. Let $G$ be a p-solvable group such that $O_{p^{\prime}}(G)=1$. Let $N$ be a normal subgroup of $G$ such that there exists an integer $l$ for which $\left[O_{p}(G),{ }_{l} N\right]=1$. Then $N \subseteq O_{p}(G)$. 
Proof. Put $V=O_{p}(G)$, and denote by $p^{r}$ the exponent of $V$. Note that $O_{p^{\prime}}(N)=1$ and put $M=O_{p^{\prime}, p}(N)$. Then by [1, Theorem 2.4],

$$
\left[V, M^{p^{r+l}}\right] \subseteq[V, M]^{p^{r+l}} \prod_{i=1}^{r+l}\left[V, p^{i} M\right]^{p^{r+l-i}} .
$$

In the previous equation, either $p^{i} \geq l$ or $r+l-i \geq r$. Therefore, $\left[V, M^{p^{r+l}}\right]=1$.

Let $H$ be a non-trivial $p^{\prime}$-Hall subgroup of $M$. Then,

$$
[V, H]=\left[V, H^{p^{r+l}}\right] \subseteq\left[V, M^{p^{r+l}}\right]=1 .
$$

Therefore, $H$ centralises $V$ and $M=H \times V$. In particular, $H \subseteq O_{p^{\prime}}(G)=1$ and therefore $O_{p^{\prime}, p}(N)=O_{p}(N)$, which implies that $N \subseteq O_{p}(G)$.

\section{REFERENCES}

1. G. A. Fernandez-Alcober, J. Gonzalez-Sanchez and A. Jaikin-Zapirain, Omega subgroups of pro-p groups, Isr. J. Math. 166 (2008), 393-412.

2. J. Gonzalez-Sanchez and T. S. Weigel, Finite p-central groups of height k, Isr. J. Math. 181 (2011), 125-143.

3. D. E. Gorenstein, Finite groups (Chelsea, New York NY, 1980).

4. P. Hall and G. Higman, The p-length of a p-solvable group and reduction theorems for Burnside's problem, Proc. London Math. Soc. 6(3) (1956), 1-42.

5. E. I. Khukhro, On p-solvable groups with a generalized p-central or powerful Sylow p-subgroup, Int. J. Group Theory 1(2) (2012, June), 51-57 (7). 